\title{
Correction to: Acute-on-chronic liver failure: consensus recommendations of the Asian Pacific association for the study of the liver (APASL): an update
}

\author{
Shiv Kumar Sarin ${ }^{1} \cdot$ Ashok Choudhury ${ }^{1} \cdot$ Manoj K. Sharma ${ }^{1} \cdot$ Rakhi Maiwall $^{1} \cdot$ Mamun Al Mahtab $^{2}$. \\ Salimur Rahman ${ }^{2}$ - Sanjiv Saigal ${ }^{3} \cdot$ Neeraj Saraf $^{3}$ - A. S. Soin ${ }^{3} \cdot$ Harshad Devarbhavi $^{4}$ - Dong Joon $\mathrm{Kim}^{5}$. \\ R. K. Dhiman ${ }^{6} \cdot$ Ajay Duseja $^{6} \cdot$ Sunil Taneja $^{6} \cdot$ C. E. Eapen ${ }^{7} \cdot$ Ashish Goel $^{7} \cdot$ Q. Ning ${ }^{8} \cdot$ Tao Chen $^{9} \cdot \mathrm{Ke} \mathrm{Ma}^{8}$. \\ Z. Duan ${ }^{9}$. Chen $\mathrm{Yu}^{9}$. Sombat Treeprasertsuk ${ }^{10}$. S. S. Hamid ${ }^{11}$. Amna S. Butt ${ }^{11}$. Wasim Jafri ${ }^{11}$ - Akash Shukla ${ }^{12}$. \\ Vivek Saraswat ${ }^{13}$. Soek Siam Tan ${ }^{14} \cdot$ Ajit Sood $^{15} \cdot$ Vandana Midha $^{15} \cdot$ Omesh Goyal ${ }^{15} \cdot$ Hasmik Ghazinyan $^{16}$. \\ Anil Arora ${ }^{17}$. Jinhua Hu ${ }^{18}$. Manoj Sahu ${ }^{19}$. P. N. Rao ${ }^{20}$. Guan H. Lee ${ }^{21}$. Seng G. Lim ${ }^{21}$. Laurentius A. Lesmana ${ }^{22}$. \\ Cosmas Rinaldi Lesmana ${ }^{22}$ - Samir Shah ${ }^{23}$. V. G. Mohan Prasad ${ }^{24}$. Diana A. Payawal ${ }^{25} \cdot$ Zaigham Abbas $^{26}$. \\ A. Kadir Dokmeci ${ }^{27}$. Jose D. Sollano ${ }^{28} \cdot$ Gian Carpio $^{28}$ - Ananta Shresta ${ }^{29}$ - G. K. Lau ${ }^{30} \cdot$ Md. Fazal Karim ${ }^{31}$. \\ Gamal Shiha ${ }^{32} \cdot$ Rino Gani $^{33} \cdot$ Kemal Fariz Kalista $^{33} \cdot$ Man-Fung Yuen $^{34} \cdot$ Seema Alam $^{35} \cdot$ Rajeev Khanna ${ }^{35}$. \\ Vikrant Sood ${ }^{35}$. Bikrant Bihari Lal ${ }^{35}$. Viniyendra Pamecha ${ }^{36}$. Ankur Jindal ${ }^{1}$ - V. Rajan ${ }^{1}$ - Vinod Arora ${ }^{1}$. \\ Osamu Yokosuka ${ }^{37}$. Madunil A. Niriella ${ }^{38} \cdot \mathrm{Hai} \mathrm{Li}^{39} \cdot \mathrm{Xiaolong} \mathrm{Qi}^{40} \cdot$ Atsushi Tanaka $^{41}$ - Satoshi Mochida ${ }^{42}$. \\ Dominic Ray Chaudhuri ${ }^{43}$. Ed Gane ${ }^{43} \cdot K_{\text {Khin Maung Win }}^{44}$. Wei Ting Chen ${ }^{45}$. Mohd. Rela ${ }^{46}$. Dharmesh Kapoor ${ }^{23}$. \\ Amit Rastogi ${ }^{3}$. Pratibha Kale ${ }^{47}$. Archana Rastogi ${ }^{48}$. Chhagan Bihari Sharma ${ }^{48} \cdot$ Meenu Bajpai $^{49} \cdot$ Virender Singh $^{6}$. \\ Madhumita Premkumar ${ }^{6}$. Sudhir Maharashi ${ }^{50}$ - A. Olithselvan ${ }^{51}$. Cyriac Abby Philips ${ }^{52}$. Anshu Srivastava ${ }^{53}$. \\ Surender K. Yachha ${ }^{53}$. Zeeshan Ahmad Wani ${ }^{54}$ - B. R. Thapa ${ }^{55}$. Anoop Saraya ${ }^{56}$. Shalimar ${ }^{56}$. Ashish Kumar ${ }^{17}$. \\ Manav Wadhawan ${ }^{57}$. Subash Gupta ${ }^{58} \cdot$ Kaushal Madan $^{59} \cdot$ Puja Sakhuja $^{60} \cdot$ Vivek Vij $^{61} \cdot$ Barjesh C. Sharma $^{62}$. \\ Hitendra Garg ${ }^{63}$. Vishal Garg ${ }^{63}$. Chetan Kalal ${ }^{64}$. Lovkesh Anand ${ }^{65}$. Tanmay Vyas ${ }^{66}$. Rajan P. Mathur ${ }^{67}$. \\ Guresh Kumar $^{68}$. Priyanka Jain ${ }^{68}$. Samba Siva Rao Pasupuleti ${ }^{68}$. Yogesh K. Chawla ${ }^{69}$. Abhijit Chowdhury ${ }^{70}$. \\ Shahinul Alam ${ }^{2}$. Do Seon Song ${ }^{71}$ - Jin Mo Yang ${ }^{71}$ - Eileen L. Yoon ${ }^{72}$. APASL ACLF Research Consortium (AARC) for \\ APASL ACLF working Party.
}

Received: 9 August 2019 / Accepted: 16 August 2019 / Published online: 9 October 2019

(c) The Author(s) 2019

\section{Correction to: Hepatology International (2019) 13:353-390 https://doi.org/10.1007/s12072-019-09946-3}

The article Acute-on-chronic liver failure: consensus recommendations of the Asian Pacific association for the study of the liver (APASL): an update, written by [Shiv Sarin], was originally published electronically on the publisher's internet portal (currently SpringerLink) on June 06, 2019 without open access.

After publication in volume 13, issue 4, page 353-390 the author decided to opt for Open Choice and to make the article an Open Access publication. Therefore, the copyright

The original article can be found online at https://doi.org/10.1007/ s12072-019-09946-3.

Shiv Kumar Sarin

sksarin@ilbs.in

Extended author information available on the last page of the article of the article has been changed to (C) The Author(s) 2019 and the article is forthwith distributed under the terms of the Creative Commons Attribution 4.0 International License (http://creativecommons.org/licenses/by/4.0/), which permits use, duplication, adaptation, distribution and reproduction in any medium or format, as long as you give appropriate credit to the original author(s) and the source, provide a link to the Creative Commons license and indicate if changes were made.

The original article has been corrected.

Open Access This article is distributed under the terms of the Creative Commons Attribution 4.0 International License (http://creativeco mmons.org/licenses/by/4.0/), which permits unrestricted use, distribution, and reproduction in any medium, provided you give appropriate credit to the original author(s) and the source, provide a link to the Creative Commons license, and indicate if changes were made. 


\section{Affiliations}

Shiv Kumar Sarin ${ }^{1} \cdot$ Ashok Choudhury $^{1} \cdot$ Manoj K. Sharma $^{1} \cdot$ Rakhi Maiwall $^{1} \cdot$ Mamun Al Mahtab $^{2}$. Salimur Rahman ${ }^{2}$ - Sanjiv Saigal ${ }^{3}$ - Neeraj Saraf ${ }^{3}$ - A. S. Soin ${ }^{3} \cdot$ Harshad Devarbhavi $^{4}$ - Dong Joon Kim $^{5}$. R. K. Dhiman ${ }^{6} \cdot$ Ajay Duseja $^{6} \cdot$ Sunil Taneja $^{6} \cdot$ C. E. Eapen ${ }^{7} \cdot$ Ashish Goel $^{7} \cdot$ Q. Ning ${ }^{8} \cdot$ Tao Chen $^{9} \cdot \mathrm{Ke} \mathrm{Ma}^{8}$. Z. Duan ${ }^{9}$. Chen $\mathrm{Yu}^{9}$. Sombat Treeprasertsuk ${ }^{10}$. S. S. Hamid ${ }^{11}$. Amna S. Butt ${ }^{11}$. Wasim Jafri ${ }^{11}$ - Akash Shukla ${ }^{12}$. Vivek Saraswat ${ }^{13}$. Soek Siam Tan ${ }^{14} \cdot$ Ajit Sood $^{15} \cdot$ Vandana Midha $^{15} \cdot$ Omesh Goyal ${ }^{15} \cdot$ Hasmik Ghazinyan $^{16}$. Anil Arora ${ }^{17}$. Jinhua Hu ${ }^{18}$. Manoj Sahu ${ }^{19}$. P. N. Rao ${ }^{20}$. Guan H. Lee ${ }^{21}$. Seng G. Lim ${ }^{21}$. Laurentius A. Lesmana ${ }^{22}$.

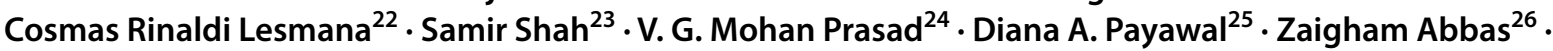
A. Kadir Dokmeci' ${ }^{27}$. Jose D. Sollano ${ }^{28} \cdot$ Gian Carpio $^{28} \cdot$ Ananta Shresta $^{29} \cdot$ G. K. Lau ${ }^{30} \cdot$ Md. Fazal Karim ${ }^{31}$. Gamal Shiha ${ }^{32} \cdot$ Rino Gani $^{33} \cdot$ Kemal Fariz Kalista $^{33} \cdot$ Man-Fung Yuen $^{34} \cdot$ Seema Alam $^{35} \cdot$ Rajeev Khanna ${ }^{35}$. Vikrant Sood ${ }^{35}$. Bikrant Bihari Lal ${ }^{35}$. Viniyendra Pamecha ${ }^{36}$. Ankur Jindal ${ }^{1}$. V. Rajan ${ }^{1}$ - Vinod Arora ${ }^{1}$.

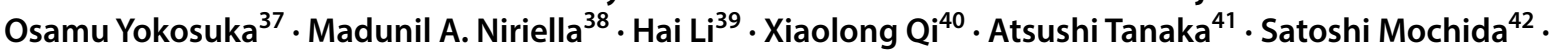
Dominic Ray Chaudhuri ${ }^{43}$. Ed Gane ${ }^{43} \cdot$ Khin Maung Win $^{44}$. Wei Ting Chen ${ }^{45}$. Mohd. Rela ${ }^{46}$. Dharmesh Kapoor ${ }^{23}$. Amit Rastogi ${ }^{3} \cdot$ Pratibha Kale $^{47} \cdot$ Archana Rastogi $^{48}$. Chhagan Bihari Sharma ${ }^{48} \cdot$ Meenu Bajpai $^{49} \cdot$ Virender Singh $^{6}$. Madhumita Premkumar ${ }^{6}$. Sudhir Maharashi ${ }^{50}$ - A. Olithselvan ${ }^{51}$. Cyriac Abby Philips ${ }^{52}$. Anshu Srivastava ${ }^{53}$. Surender K. Yachha ${ }^{53}$. Zeeshan Ahmad Wani ${ }^{54}$ - B. R. Thapa ${ }^{55}$. Anoop Saraya ${ }^{56}$. Shalimar ${ }^{56}$. Ashish Kumar ${ }^{17}$. Manav Wadhawan ${ }^{57}$. Subash Gupta ${ }^{58} \cdot$ Kaushal Madan $^{59}$. Puja Sakhuja ${ }^{60} \cdot$ Vivek Vij $^{61} \cdot$ Barjesh C. Sharma $^{62}$. Hitendra Garg ${ }^{63}$. Vishal Garg ${ }^{63}$. Chetan Kalal ${ }^{64}$. Lovkesh Anand ${ }^{65}$. Tanmay Vyas ${ }^{66}$. Rajan P. Mathur ${ }^{67}$. Guresh Kumar $^{68}$. Priyanka Jain ${ }^{68}$. Samba Siva Rao Pasupuleti ${ }^{68}$. Yogesh K. Chawla ${ }^{69}$. Abhijit Chowdhury ${ }^{70}$. Shahinul Alam ${ }^{2}$. Do Seon Song ${ }^{71}$. Jin Mo Yang ${ }^{71}$. Eileen L. Yoon ${ }^{72}$. APASL ACLF Research Consortium (AARC) for APASL ACLF working Party.

1 Department of Hepatology, Institute of Liver and Biliary Sciences, New Delhi 110070, India

2 Department of Hepatology, Bangabandhu Sheikh Mujib Medical University, Dhaka, Bangladesh

3 Department of Hepatology, Medanta The Medicity, Gurgaon, India

4 Department of Hepatology, St John Medical College, Bangalore, India

5 Department of Internal Medicine, Hallym University College of Medicine, Seoul, South Korea

6 Department of Hepatology, PGIMER, Chandigarh, India

7 Department of Hepatology, CMC, Vellore, India

8 Institute and Department of Infectious Disease, Tongji Hospital, Tongji Medical College, Huazhong University of Science and Technology, Wuhan, China

9 Translational Hepatology Institute Capital Medical University, Beijing You'an Hospital, Beijing, China

10 Department of Medicine, Chulalongkorn University, Bangkok, Thailand

11 Department of Medicine, Aga Khan University Hospital, Karachi, Pakistan

12 Department of Gastroenterology, Lokmanya Tilak Municipal General Hospital and Lokmanya Tilak Municipal Medical College, Sion, Mumbai, India

13 Department of Gastroenterology, SGPGIMS, Lucknow, India

14 Department of Medicine, Hospital Selayang, Bata Caves, Selangor, Malaysia

15 Department of Gastroenterology, DMC, Ludhiana, India

16 Department of Hepatology, Nork Clinical Hospital of Infectious Disease, Yerevan, Armenia
17 Department of Gastroenterology and Hepatology, Sir Ganga Ram Hospital and GRIPMER, New Delhi, Delhi, India

18 Department of Medicine, 302 Millitary Hospital, Beijing, China

19 Department of Gastroenterology and Hepatology Sciences, IMS \& SUM Hospital, Bhubaneswar, Odisha, India

20 Asian Institute of Gastroenterology, Hyderabad, India

21 Division of Gastroenterology and Hepatology, Department of Medicine, National University Health System, Singapore, Singapore

22 Digestive Disease and GI Oncology Centre, Medistra Hospital, Jakarta, Indonesia

23 Department of Hepatology, Global Hospitals, Mumbai, India

24 Department of Gastroenterology, VGM Hospital, Coimbatore, India

25 Fatima University Medical Center Manila, Manila, Philippines

26 Department of Medicine, Ziauddin University Hospital, Karachi, Pakistan

27 Department of Medicine, Ankara University School of Medicine, Ankara, Turkey

28 Department of Medicine, University of Santo Tomas, Manila, Philippines

29 Department of Hepatology, Foundation Nepal Sitapaila Height, Kathmandu, Nepal

30 Department of Medicine, Humanity and Health Medical Group, New Kowloon, Hong Kong, China

31 Department of Hepatology, Sir Salimullah Medical College, Dhaka, Bangladesh

32 Egyptian Liver Research Institute And Hospital, Cairo, Egypt 
33 Division of Hepatobiliary, Department of Internal Medicine, Faculty of Medicine, Cipto Mangunkusumo Hospital, Universitas Indonesia, Jakarta, Indonesia

34 Department of Medicine, Queen Mary Hospital Hong Kong, The University of Hong Kong, Hong Kong, China

35 Department of Pediatric Hepatology, Institute of Liver and Biliary Sciences, New Delhi, Delhi, India

36 Department of Hepatobilliary Pancreatic Surgery and Liver Transplant, Institute of Liver and Biliary Sciences, New Delhi, Delhi, India

37 Professor Emeritus, Chiba University, Chiba, Japan

38 Department of Medicine, University of Kelaniya, Ragama, Sri Lanka

39 Department of Gastroenterology, Ren Ji Hospital, School of Medicine, Shanghai Jiao Tong University, Shanghai, China

40 CHESS Frontier Center, The First Hospital of Lanzhou University, Lanzhou University, Lanzhou, China

41 Department of Medicine, Tokyo University School of Medicine, Tokyo, Japan

42 Department of Gastroenterology and Hepatology, Faculty of Medicine, Saitama Medical University, Saitama, Japan

43 New Zealand Liver Transplant Unit, Auckland Hospital, Auckland, New Zealand

44 Yangon GI and Liver Centre, Pabedan, Yangon, Myanmar

45 Division of Hepatology, Department of Gastroenterology and Hepatology, Chang Gung Medical Foundation, Linkou Chang Gung Memorial Hospital, Taoyuan, Taiwan

46 Department of Liver Transplant Surgery, Dr. Rela Institute and Medical Centre, Chennai, India

47 Department of Microbiology, Institute of Liver and Biliary Sciences, New Delhi, Delhi, India

48 Department of Pathology, Institute of Liver and Biliary Sciences, New Delhi, Delhi, India

49 Department of Immunohematology and Transfusion Medicine, Institute of Liver and Biliary Sciences, New Delhi, Delhi, India

50 Department of Gatroenterology, SMS Med College, Jaipur, India

51 Division of Liver Transplantation and Hepatology, Manipal Hospitals, Bangalore, India

52 The Liver Unit, Cochin Gastroenterology Group, Ernakulam Medical Centre, Kochi, India
53 Department of Pediatric Gastroenterology, SGPGIMS, Lucknow, India

54 Noora Hospital in Srinagar, Shrinagar, India

55 Department of Gastroenterology and Pediatric Gastroenterology, PGIMER, Chandigarh, India

56 Department of Gastroenterology and Human Nutrition, AIIMS, New Delhi, India

57 Department of Gastroenterology, Hepatology and Liver Transplant, B L K Hospital, New Delhi, India

58 Centre for Liver and Biliary Science, Max Hospital, New Delhi, India

59 Department of Gastroenterology, Hepatology and Liver Transplant, Max Hospital, New Delhi, India

60 Department of Pathology, GB Pant Hospital, New Delhi, India

61 Department of Liver Transplant and Hepatobilliary Surgery, Fortis Hospital, New Delhi, India

62 Department of Gastroenterology, GB Pant Hospital, New Delhi, India

63 Department of Gastroenterology, Hepatology and Liver Transplant, Apollo Hospital, New Delhi, India

64 Department of Hepatology, Sir H N Reliance Hospital and Research Centre, Mumbai, India

65 Department of Gastroenterology and Hepatology, Narayana Hospital, Gurugram, India

66 Department of Hepatology, Parimal Multi-Speciality Hospital, Ahmedabad, India

67 Department of Nephrology, Institute of Liver and Biliary Sciences, New Delhi, India

68 Department of Statistics and Clinical Research, Institute of Liver and Biliary Sciences, New Delhi, India

69 Department of Hepatology and Gastroenterology, Kalinga Institute of Med Sciences, KIIT University, Bhubaneswar, India

70 Department of Hepatology, Institute of Post Graduate Medical Education and Research, Kolkata, India

71 Department of Internal Medicine, College of Medicine, The Catholic University of Korea, Seoul, South Korea

72 Department Of Internal Medicine, Inje University College of Medicine, Busan, South Korea 